\title{
Monitoring the Ecological Succession and Regeneration Status of a Post-Remediated Hydrocarbon Impacted Site in Parts of Ahia Oil-Field, in Omudioga, EMOLGA, Rivers State, Nigeria
}

\author{
${ }^{1}$ E.A.B. Edu, ${ }^{2}$ N.L. Edwin-Wosu and ${ }^{2}$ N.D. Obiechere \\ ${ }^{1}$ Department of Botany, University of Calabar, Calabar, P.M.B. 1115, Nigeria \\ ${ }^{2}$ Department of Plant Science and Biotechnology, Faculty of Biological Science, College of Natural and \\ Applied Sciences, University of Port Harcourt, Choba, P.M.B. 5323, Port Harcourt
}

\begin{abstract}
Background: Several biotic and abiotic factors including the influence of hydrocarbon and inorganic activities on population structure and regeneration of vegetation have been noted as agents of species on ecological succession. Similar studies have also been carried out by many workers in different forest ecosystem, but no information has been generated on this aspect for the post remediated Ahia oil-field under local environmental condition in Rivers State, Nigeria. Objective: Therefore, this study was aimed at assessing the post-phytodiversity and regeneration status of post-remediated crude oil impacted site in Ahia oil-field, in Rivers State, Nigeria. Methodology: Despite the heterogeneous mosaic nature of secondary vegetation of the study area, a total representative of 67 species under 15 families are recorded with monotypic heterogeneity of life forms in the study site, with various diversity and abundance. The Poaceae among the prevalent dominant families was the most diverse in species richness. The total representative species were establishing through coppicing, seedling and rhizome with greater percentage $(42=63 \%)$ of the species exhibiting multiplier mode while $25=37.31 \%$ were solitary in regeneration status. Results: The phytosociological index assessment shows that Oldenlandia herbacea, Axonopus compressus and Heterotis rotundifolia had recorded the highest frequency of $50=4.65 \%$ occurrence, respectively. Axonopus compressus further had the highest abundance of $100=6.19 \%$, Importance Value Index of $11.95 \%$, richness of 0.06 and evenness of 0.03 in species diversity. The spatial distribution of species at the study site recorded a contiguous pattern with Oldenlandia herbacea recording the highest distribution pattern. The increase in the herbaceous life form of the study site is an indication of a primary regenerative succession that tends towards a pioneer vegetation array of shrubby and tree species of climax status. Conclusion: The study, therefore provide a complete view of regeneration trend in the study area, which is rich in regenerating species that could result in establishment of a diverse natural forest if protected with special attention to the scope of biodiversity conservation priority.
\end{abstract}

Key words: Abundance, species diversity, frequency, density, importance value index

Insight Ecology 4 (1): 24-34, 2015

\section{INTRODUCTION}

Vegetation in man made habitat involving studies on changes in species diversity and floristic composition of habitats have received appreciable attention ${ }^{1-4}$. Several factors are known to contribute to changes in species diversity and composition at any given time and place. Recently, man has faced one of the most horrible

Corresponding Author: N.L. Edwin-Wosu, Department of Plant Science and Biotechnology, Faculty of Biological Science, College of Natural and Applied Sciences, University of Port Harcourt, Choba, P.M.B. 5323, Port Harcourt ecological crises such as pollution of the environment which sometimes in the past was pure, virgin, undisturbed, uncontaminated and basically quite hospitable for him. Crude oil exploration is a double edge sword as could be reaffirmed by the fact that while it improves on societal development it has a colossal impact on the people and the environment including plant species ${ }^{5}$. Kinako ${ }^{6}$, reported on the effects of oil pollution on species number and productivity in a terrestrial ecosystem. This corroborates the slow destruction of vegetation and agricultural land by oil spill which occur during petroleum operation ${ }^{7}$. 
Several other biotic and abiotic factors have been noted as agents of species ecological amplitude ${ }^{8-10}$. This concomitantly amount to some degree of ecological succession causing direct changes in plant species abundance in terms of frequency of occurrence and density. Other succession impacts involve changes in species diversity (in terms of richness and evenness), relative density, relative frequency, relative abundance, Importance Value Index (IVI), ratio of abundance to frequency $(\mathrm{A} / \mathrm{F})$ and plant species regeneration and habit. Ecological succession is a phenomenon or process by which a community progressively transforms itself until a stable community is formed. It is a fundamental concept in ecology and refers to more or less predictable and orderly changes in the composition and structure of an ecological community. Succession may be initiated either by some formation of new, unoccupied habitat or by some form of disturbance of an existing community. Several works on ecological succession of diverse plant species assemblage involving both rare and invasive species on disturbed sites have been documented $^{11,12}$.

In ecosystems, abiotic disturbance and stressful conditions induce profound modifications on the landscape and plant communities. It is thus difficult to estimate the rate or degree of spontaneous vegetation recolonization or regeneration. Regeneration is a critical phase of forest management, because it maintains the desired species composition and stocking after disturbance $^{13}$. This assertion corroborates the floristic and diversity trend of plant species regeneration in impacted sites in parts of southern Nigeria ${ }^{3,4}$. Various other studies of the influence of hydrocarbon and inorganic activities on population structure and regeneration of vegetation have been carried out by many workers in different forest ecosystem ${ }^{14}$. However, no information has been generated on this aspect for the post remediated Ahia oil-field under the local environment. This study was aimed at assessing the post-phytodiversity and regeneration status of the postremediated crude oil impacted site.

\section{MATERIALS AND METHODS}

Geomorphological description of the study area/site: The study area (Omudioga) is a tropical rainforest, one of the centres of agricultural zones situated between Lat. 6 43 '30' $\mathrm{N}$ and Lat. 6 54'0" $\mathrm{N}$ and Long. 5'0'0"E and Long. 5'10'0"E in Emohua Local Government Area of Rivers State (Fig. 1 and 2). It is a tropical climate within the equatorial climate region characterized by maximum rainfall, relative humidity and maximum temperature. The edaphic and topographic formation show that the study area is a Table and is characterized by sandy-loam soil. The vegetation is of typical secondary nature with its prevalent species of ecological succession regenerating from the effect of seriously impacted oil spill and post remediation process. The successive vegetation of the area is characterized by prevalent species of shrubs, herbs, climbers, lianers and dominantly members of Poaceae, Asteraceae, Sterculiaceae, Fabaceae, Tiliaceae, Malvaceae, Rubiaceae, Melastomataceae, Euphorbiaceae, Cyperaceae, Commelinaceae, Lamiaceae, Onagraceae, Amaranthaceae and Scrophulariaceae plant families, typical of secondary succession fallowed bush. The environment has also witnessed some form of human activities such as farming, domestic wood logging and bush burning far and near residential areas by the local communities leaving fields of plantations such as Manihot spp. Musa spp. and Zea mays Though originally a virgin forest of various heterogeneous vegetation structures, the effect of these human activities, impact of post oil spill and post remediation has consequently left it with some form of irregular vegetation features. This can therefore be categorized as a low land secondary mosaic forest as described by Hopkin ${ }^{15}$. However, the vegetation is still described as rainforest vegetation in relation to similar view of vegetation analysis by $\mathrm{SAF}^{16}$ and Edwin-Wosu ${ }^{17-20}$.

Vegetation assessment: It was carried out by adopting the simple random sampling based on standard procedures for ecological assessment studies ${ }^{21}$ with specific quadrate direction of $100 \times 50 \mathrm{~m}^{2}$ to determine the regeneration status of the site. This was divided into sampling unit to give a total of five quadrate sampling plot of $20 \times 50 \mathrm{~m}$ each and was systematically sampled. All the important representative plant species sampled were identified in the field as far as possible and were properly authenticated using flora such as described in previous studies $^{22-28}$.

Data analyses: The frequency of distribution, abundance and density of the representative species of the study site were estimated using the methods of Austin and Greig-Smith ${ }^{29}$, Kershaw ${ }^{30}$ and Shukla and Chandal $^{31}$ approach as modified in Bonham ${ }^{32}$. The species diversity and dominance over the study site was estimated using the Shannon and Weaver index ${ }^{33}$. Vegetation was described in semi-quantitative terms ${ }^{34}$ and in accordance species with a wide frequency of distribution with many stands are described as very 


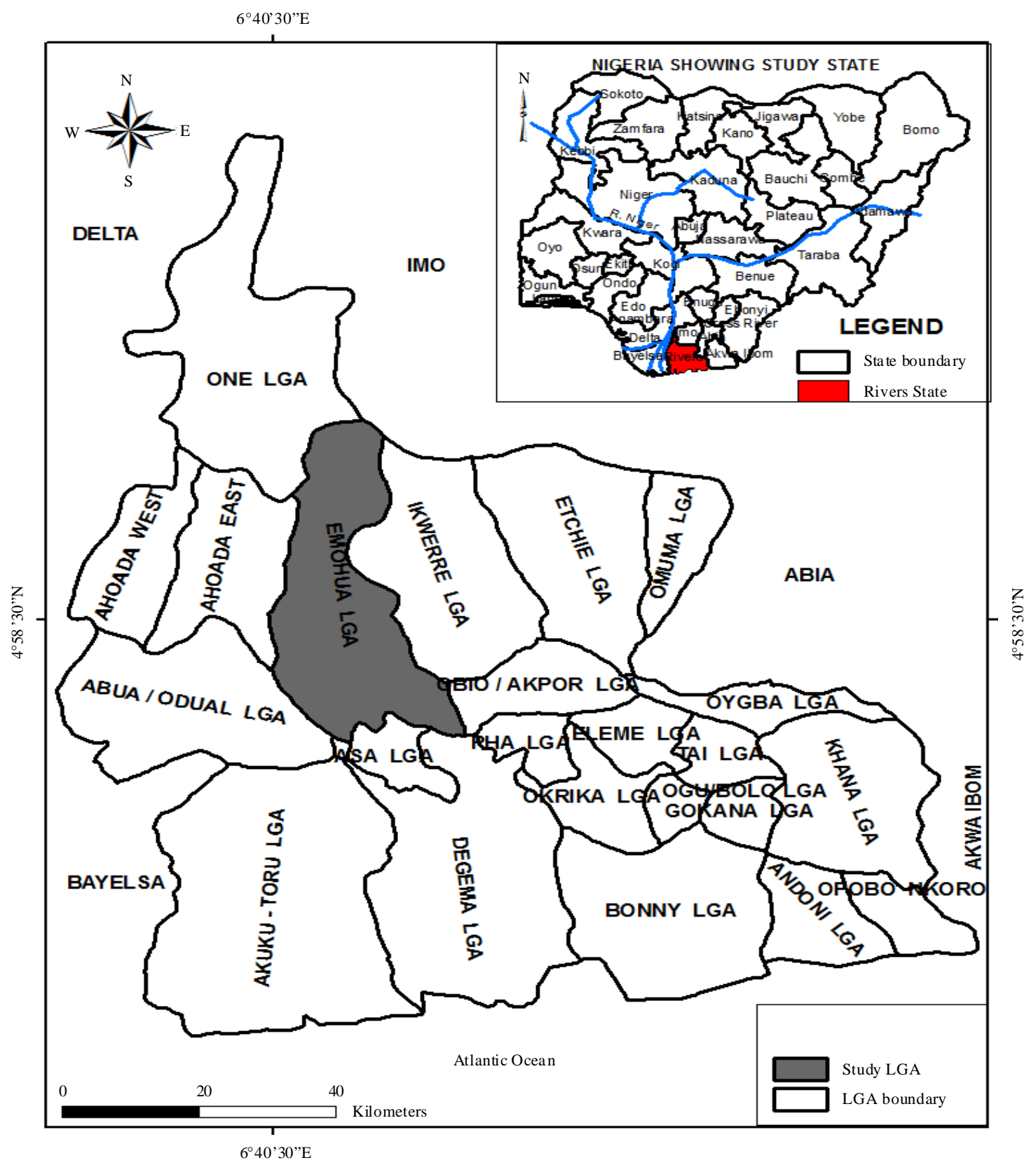

Fig. 1: Map of River States showing study area (Emohua)

abundant $(++++)$. Some species with similarly wide frequency of distribution but with few stands are said to be less frequent, abundant or restricted species $(+++)$. The species with limited geographical distribution and with a few stands are termed scarce or occasional $(++)$ and very scarce or rare $(+)$ species. The species designated $(++)$ and $(+)$ are often seen as being prone to elimination due to their limited extent alone beside any other factors. Relative density, relative abundance and relative frequency were estimated following method $^{35}$, while the Importance Value Index (IVI) was estimated by the cumulative ecological indices using the Shukla and Chandal method ${ }^{31}$. The ratio of abundance to frequency for different species was determined for distribution patterns. Thus with the "Thumb of rule" designated as follows: Regular $(<0.03)$, Random $(0.03-0.05)$ and Contiguous $(>0.05)$ distribution as adopted by Curtis and Cottam ${ }^{36}$. 


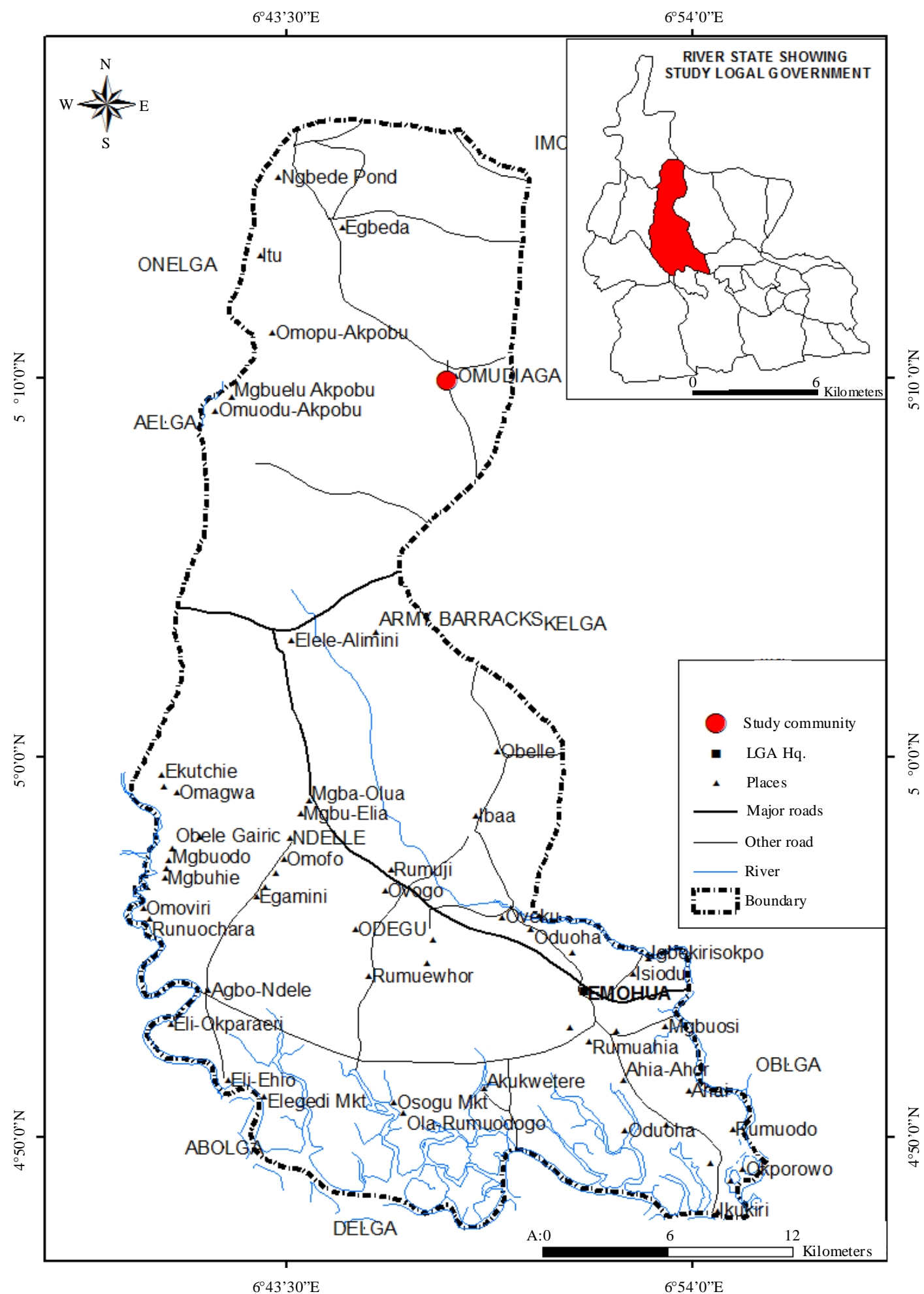

Fig. 2: Map of Emohua (study area) showing study site 
Table 1: List of representative species in parts of Ahia Oil-Field in Omudioga, EMOLGA, Rivers State

\begin{tabular}{|c|c|c|c|c|c|c|}
\hline Species & $\mathrm{F}(\%)$ & Family & Habit & Mode of regeneration & Common names & Remarks \\
\hline Panicum laxum SW & 100 & Poaceae & Herb & $\mathrm{R} / \mathrm{C}$ & Grass & ++++ \\
\hline Melochia pyramidata Linn & 100 & Sterculiaceae & Herb & $\mathrm{R}$ & NA & ++++ \\
\hline Euphorbia prostrata Linn & 100 & Euphorbiaceae & Herb & $\mathrm{S} / \mathrm{C}$ & NA & ++++ \\
\hline Mimosa invisa Mart. & 75 & Fabaceae-Mimo. & Herb & $\mathrm{S} / \mathrm{C}$ & Giant sensitive plant & +++ \\
\hline Mimosa pudica Linn. & 100 & Fabaceae-Mimo. & Herb & $\mathrm{S} / \mathrm{C}$ & Sensitive plant & ++++ \\
\hline Commelina erecta Linn & 50 & Commelinaceae & Herb & $\mathrm{R} / \mathrm{C}$ & White mouth day flower & ++ \\
\hline Commelina benghalensis Linn & 50 & Commelinaceae & Herb & $\mathrm{R} / \mathrm{C}$ & NA & ++ \\
\hline Chrysopon aciculatus (Retz) Trin. & 50 & Poaceae & Herb & $\mathrm{S} / \mathrm{R}$ & NA & ++ \\
\hline Panicum maximum Jaeq. & 100 & Poaceae & Herb & $\mathrm{R} / \mathrm{C}$ & Grass & ++++ \\
\hline Panicum repens Linn. & 50 & Poaceae & Herb & $\mathrm{R} / \mathrm{C}$ & Torpedo grass & ++ \\
\hline Spermacoce ocymoides Burm f & 50 & Rubiaceae & Herb & S & NA & ++ \\
\hline Platostoma africanum P. Beauv. & 50 & Lamiaceae & Herb & $\mathrm{S} / \mathrm{C}$ & NA & ++ \\
\hline Eleusine indica Geartn. & 100 & Poaceae & Herb & $\mathrm{S} / \mathrm{R}$ & Bull grass & ++++ \\
\hline Ludiwigia decurens Walt. & 50 & Onagraceae & Herb & $\mathrm{S} / \mathrm{C}$ & Water primrose & ++ \\
\hline Ludiwigia decurencs Walt. & 100 & Onagraceae & Herb & $\mathrm{S} / \mathrm{C}$ & Water primrose & ++++ \\
\hline Kyllinga pumilia Michx. & 100 & Cyperaceae & Herb & $\mathrm{R} / \mathrm{S}$ & Sedge & ++++ \\
\hline Kyllinga sequamulata Thonn. ex vahl. & 50 & Cyperaceae & Herb & $\mathrm{R} / \mathrm{S}$ & Sedge & ++ \\
\hline Veronica cinerea (Linn) Less. & 75 & Asteraceae & Herb & $\mathrm{S} / \mathrm{C}$ & Little iron weed & +++ \\
\hline Mariscus longibracteatus Cherm & 50 & Cyperaceae & Herb & $\mathrm{S} / \mathrm{C}$ & Sedge & ++ \\
\hline Calopogonium mucunoides Desv. & 100 & Fabaceae-papi & Herb & $\mathrm{S} / \mathrm{R}$ & Dead man's yam bean & ++++ \\
\hline Mitracarpus vilosus SW & 50 & Rubiaceae & Herb & $\mathrm{S}$ & NA & ++ \\
\hline Pennisetum violaceum (Lam) L. Rich. & 75 & Poaceae & Herb & $\mathrm{S} / \mathrm{R}$ & Grass & +++ \\
\hline Solenostemo monstachyus (P. Beauv) & 75 & Lamiaceae & Herb & $\mathrm{S} / \mathrm{C}$ & NA & +++ \\
\hline Asystasia gagentica (Linn) T. Anders & 100 & Asteraceae & Herb & $\mathrm{S} / \mathrm{C}$ & NA & ++++ \\
\hline Alternanthera pungens H.B.and K & 50 & Amaranthaceae & Herb & $\mathrm{S} / \mathrm{C}$ & Khakiweed & ++ \\
\hline $\begin{array}{l}\text { Eragrostic tenella (Linn) P. Beauv. ex Poem } \\
\text { and Schult }\end{array}$ & 100 & Poaceae & Herb & $\mathrm{S} / \mathrm{R}$ & Grass & ++++ \\
\hline Perotis indica (Linn) O. Ktze & 50 & Poaceae & Herb & $\mathrm{R}$ & NA & ++ \\
\hline Paspalum conjugatum Berg & 50 & Poaceae & Herb & $\mathrm{R} / \mathrm{C}$ & Sour grass & ++ \\
\hline Oplismenus burmanii (Retz) P. Beauv & 50 & Poaceae & Herb & $\mathrm{R}$ & NA & ++ \\
\hline Pentodon pentandrus Schum and Thonn & 50 & Rubiaceae & Herb & $\mathrm{S} / \mathrm{C}$ & NA & ++ \\
\hline Kyllinga bulbosa Beauv. & 50 & Cyperaceae & Herb & $\mathrm{S} / \mathrm{R}$ & Sedge & ++ \\
\hline Gomphrena celosioides Mart. & 50 & Amaranthaceae & Herb & $\mathrm{S} / \mathrm{R}$ & NA & ++ \\
\hline Spilanthes filicaulis Schum and Thonn & 100 & Asteraceae & Herb & $\mathrm{S}$ & NA & ++++ \\
\hline Imperata cylindrical Linn. & 50 & Poaceae & Herb & $\mathrm{S} / \mathrm{C}$ & Congo grass & ++ \\
\hline Spermacoce verticillata Linn. & 75 & Rubiaceae & Herb & $\mathrm{S}$ & NA & +++ \\
\hline Oldenlandia herbacea (Linn) Roxb. & 50 & Rubiaceae & Herb & $\mathrm{S}$ & NA & ++ \\
\hline Diodia scandens SW & 50 & Rubiaceae & Herb & $\mathrm{S}$ & NA & ++ \\
\hline Eragrostis ciliaria (Linn) R.B. & 50 & Poaceae & Herb & $\mathrm{S}$ & Love grass & ++ \\
\hline Digitria horizontalis Willd. & 50 & Poaceae & Herb & $\mathrm{S}$ & Crab grass & ++ \\
\hline Chloris pilosa Schumach & 100 & Poaceae & Herb & $\mathrm{S}$ & Grass & ++++ \\
\hline Phyllantus nururoides Mull-Arg. & 75 & Euphorbiaceae & Herb & S & Gale of the wind & +++ \\
\hline Triumfetta cordifolia A. Rich. & 50 & Tiliaceae & Herb & $\mathrm{S} / \mathrm{C}$ & NA & ++ \\
\hline Axonopus compressus (SW) P. Beauv. & 100 & Poaceae & Herb & $\mathrm{R}$ & Carpet grass & ++++ \\
\hline Eclipta alba (L.) Hassk & 50 & Asteraceae & Herb & $\mathrm{R}$ & NA & ++ \\
\hline Fimbristylis littovalis Gaudet & 50 & Cyperaceae & Herb & $\mathrm{S}$ & NA & ++ \\
\hline Paspalum scrobiculatum Linn & 50 & Poaceae & Herb & $\mathrm{R}$ & Rice grass & ++ \\
\hline Desmodium triflorum (Linn) DC & 75 & Fabaceae-papilio & Herb & $\mathrm{R}$ & Three flower tickrefoll & +++ \\
\hline Scoparia dulcis Linn & 75 & Scrophulariaceae & Herb & $\mathrm{S}$ & Sweet broom weed & +++ \\
\hline Chamaecrista mimosoides (L.) Greene. & 50 & Fabaceae-ceasal & Herb & $\mathrm{S} / \mathrm{C}$ & Tea senna & ++ \\
\hline Sporobulus pyramidalis P. Beauv. & 50 & Poaceae & Herb & $\mathrm{S}$ & Cat's tail grass & ++ \\
\hline Bidens pilosa Linn. & 50 & Asteraceae & Herb & S & Black jack & ++ \\
\hline Cyperus difformis Linn. & 75 & Cyperaceae & Herb & $\mathrm{S} / \mathrm{R}$ & Sedge & +++ \\
\hline Cyperus esculentus Linn & 50 & Cyperaceae & Herb & $\mathrm{S} / \mathrm{R}$ & Yellow nut sedge & ++ \\
\hline Cyperus haspan Linn. & 75 & Cyperaceae & Herb & $\mathrm{S} / \mathrm{R}$ & Sedge & +++ \\
\hline Cyperus longibracteatus Cherm. & 75 & Cyperaceae & Herb & $\mathrm{S} / \mathrm{R}$ & Sedge & +++ \\
\hline Melochia mellisifolia (Sm) Jacq & 50 & Sterculiaceae & Herb & $\mathrm{R}$ & NA & ++ \\
\hline Chromoleana odorata (L.) R.M & 75 & Asteraceae & Herb & $\mathrm{S} / \mathrm{R} / \mathrm{C}$ & Siam weed & +++ \\
\hline \multicolumn{7}{|l|}{ King and Robinson } \\
\hline Desmodium Linn. (Sw) Desv. & 50 & Papilionioideae & Herb & $\mathrm{S} / \mathrm{C}$ & Beggar weed & ++ \\
\hline Oldenlandia corymbosa Linn. & 100 & Rubiaceae & Herb & S & NA & ++++ \\
\hline Phyllantus amarus Schum and Thonn & 50 & Euphorbiaceae & Herb & $\mathrm{S}$ & Stone breaker & ++ \\
\hline
\end{tabular}


Table 1: Continue

\begin{tabular}{|c|c|c|c|c|c|c|}
\hline Species & $\mathrm{F}(\%)$ & Family & Habit & Mode of regeneration & Common names & Remarks \\
\hline Sida acuta Burm. F & 50 & Malvaceae & Herb & $\mathrm{S} / \mathrm{C}$ & Broom weed & ++ \\
\hline Sida cordifolia Linn & 50 & Malvaceae & Herb & $\mathrm{S} / \mathrm{C}$ & Lima & ++ \\
\hline Malvastrum coromandelianum (Linn) Garck & 50 & Malvaceae & Herb & $\mathrm{S} / \mathrm{C}$ & NA & ++ \\
\hline Sida corymbosa R. E. Fries. & 50 & Malvaceae & Herb & $\mathrm{S} / \mathrm{C}$ & Wire weed & ++ \\
\hline Centrosema pubescens Benth & 100 & Fabaceae & Herb & S & NA & ++++ \\
\hline Pycreus lanceolatus (Pior) C.B.Cl. & 75 & Cyperaceae & Herb & $\mathrm{S} / \mathrm{R}$ & Dumb cane & +++ \\
\hline Heterotis rotundifolia (Sm) Jacq. & 75 & Melastomataceae & Herb & $\mathrm{S} / \mathrm{R}$ & Rock rose (cheek weed) & +++ \\
\hline
\end{tabular}

\section{RESULT}

The Ahia oil-field (among the marginal oil field) in part of the study area is a secondary vegetation, heterogeneous and mosaic in nature. The heterogeneity is a monotypic complex of herbaceous life forms involving mainly herbs during the study period. Despite the heterogeneous mosaic monotypic habit of the study site there existed arrays of plant species in various diversity and abundance with representative species recorded in Table 1 . The study site secondary vegetation of the area is being characterized with both biotic and abiotic influences. This is being attributed to a number of retrogressive processes such as the influence of post oil spill incident, post-remediation processes, the regeneration and floristic succession of the study site coupled with seasonal variation under local environmental conditions. This resulted to total change in the vegetation array in terms of structure, habit, abundance and diversity.

The vegetation complex recorded a total of 67 representative plant species under 15 families with monotypic heterogeneity of life forms. Seven families (Poaceae, Euphorbiaceae, Fabaceae, Rubiaceae, Cyperaceae, Asteraceae and Malvaceae) were prevalently dominant with one (Euphorbiaceae) in abundance while the above mentioned six species were very abundant. Under such ecological assessment, all the representative species were mainly herbs at the period of study distributed among 15 families. Among the prevalent families, Poaceae was the most diverse in richness with 17 species followed by Cyperaceae ${ }^{10}$ while Fabaceae and Rubiaceae had 7 species, respectively, Asteraceae ${ }^{6}$ and Malvaceae ${ }^{4}$ species among the representative samples.

Similar study based on phytosociological and quantitative index of analysis on species prevalence of the study site (Table 2) shows that 16 species have recorded the highest frequency $(100=2.22 \%)$ of occurrence among the representative, while 43 species had maximum density value range of $10-50=0.93-4.65 \%$ with the highest value of
$50=4.65 \%$ recorded among Oldenlandia herbacea, Axonopus compressus (carpet grass) and Heterotis rotundifolia (chick weed), respectively. A total representative of 58 species had maximum abundance with value range of $10-100=0.62-6.19 \%$ and carpet grass having the highest value of $100=6.19 \%$ which also recorded the highest status of species prevalence with Importance Value Index (IVI) of $11.95 \%$ and diversity of 0.06 richness and 0.03 evenness. The ratio of abundance to frequency showed that the species of the study site is generally contiguous in distribution pattern with Oldenlandia herbacea recording the highest distribution is spatial continuum. Generally, in all bounding coordinates there was increase in herbaceous status with all representative species exhibiting as herbs in life forms. Such herbaceous increase is an indication of a primary regenerative succession which seems to be progressive toward climax vegetation of shrubby and tree species if protected with special attention to the scope of biodiversity conservation priority. The study has shown that open canopy may favour germination and seedling establishment through increased solar radiation, incident on the forest floor. New species were found regenerating, which were absent as adults. Result have also shown that the species in their diverse potential have re-established through various mode of regeneration status with greater percentage $(42=63 \%)$ of the species exhibiting multiplier mode of regeneration while $25=37.31 \%$ were solitary in regeneration status. The species were establishing through coppering, rhizome and seedling. However, 17 species exhibited a single mode of regeneration by seedling, eight species by rhizome while the rest exhibited multiplier mode of regeneration with six species via rhizome/coppicing, 20 in rhizome/coppicing, 15 in rhizome/seedling and 1 in seedling/rhizome/ coppicing. Though, the site was crude oil impacted and post-remediated, the regeneration of many indigenous species was evident and this is very important for biodiversity conservation. 
Table 2: Quantitative list of representative species in parts of Ahia Oil-Field in Omudioga, EMOLGA, Rivers State

\begin{tabular}{|c|c|c|c|c|c|c|c|c|c|c|}
\hline Species & $\mathrm{F}(\%)$ & $\mathrm{D}$ & A & $\mathrm{RF}(\%)$ & $\mathrm{RD}(\%)$ & RA (\%) & IVI & SDR & SDE & $\mathrm{A} / \mathrm{F}$ \\
\hline Panicum laxum SW & 100 & 15.00 & 15.0 & 2.22 & 1.40 & 0.93 & 4.55 & 0.03 & 0.02 & 0.15 \\
\hline Melochia pyramidata Linn & 100 & 20.00 & 20.0 & 2.22 & 1.86 & 1.24 & 5.32 & 0.03 & 0.02 & 0.20 \\
\hline Euphorbia prostrata Linn & 100 & 15.00 & 25.0 & 2.22 & 2.33 & 1.55 & 6.10 & 0.03 & 0.02 & 0.25 \\
\hline Mimosa invisa Mart. & 75 & 30.00 & 40.0 & 1.67 & 2.79 & 2.48 & 6.49 & 0.04 & 0.02 & 0.05 \\
\hline Mimosa pudica Linn. & 100 & 25.00 & 25.0 & 2.22 & 2.33 & 1.55 & 6.10 & 0.03 & 0.02 & 0.25 \\
\hline Commelina eracta Linn & 50 & 12.50 & 25.0 & 1.11 & 1.16 & 1.55 & 3.82 & 0.02 & 0.01 & 0.50 \\
\hline Commelina benghalensis Linn & 50 & 7.50 & 15.0 & 1.11 & 0.70 & 0.93 & 2.74 & 0.02 & 0.01 & 0.30 \\
\hline Chrysopon aciculatus (Retz) Trin. & 50 & 15.00 & 30.0 & 1.11 & 1.40 & 1.86 & 4.37 & 0.03 & 0.02 & 0.60 \\
\hline Panicum maximum Jaeq. & 100 & 20.00 & 20.0 & 2.22 & 1.86 & 1.24 & 5.32 & 0.03 & 0.02 & 0.20 \\
\hline Panicum repens Linn. & 50 & 10.00 & 20.0 & 1.11 & 0.93 & 1.24 & 3.28 & 0.02 & 0.01 & 0.40 \\
\hline Spermacoce ocymoides Burm f & 50 & 15.00 & 30.0 & 1.11 & 1.40 & 1.86 & 4.37 & 0.02 & 0.02 & 0.30 \\
\hline Platostoma africanum P. Beauv. & 50 & 8.75 & 17.5 & 1.11 & 0.81 & 1.08 & 3.00 & 0.02 & 0.01 & 0.35 \\
\hline Eleusine indica Geartn. & 100 & 37.50 & 37.5 & 2.22 & 3.49 & 2.32 & 8.03 & 0.04 & 0.02 & 0.38 \\
\hline Ludiwigia decurens Walt. & 50 & 10.00 & 20.0 & 1.11 & 0.93 & 1.24 & 3.28 & 0.02 & 0.01 & 0.40 \\
\hline Ludiwigia decurencs Walt. & 100 & 5.00 & 5.0 & 2.22 & 0.47 & 0.31 & 3.00 & 0.02 & 0.01 & 0.05 \\
\hline Kyllinga pumilia Michx. & 100 & 32.50 & 32.5 & 2.22 & 3.02 & 2.01 & 7.25 & 0.04 & 0.02 & 0.33 \\
\hline Kyllinga sequamulata Thonn. ex vahl. & 50 & 25.00 & 50.0 & 1.11 & 2.33 & 3.10 & 6.54 & 0.04 & 0.02 & 1.00 \\
\hline Veronica cinerea (Linn) Less. & 75 & 7.50 & 10.0 & 1.67 & 0.70 & 0.62 & 2.99 & 0.02 & 0.01 & 0.13 \\
\hline Mariscus longibracteatus Cherm & 50 & 11.25 & 22.5 & 1.11 & 1.05 & 1.39 & 3.55 & 0.02 & 0.01 & 0.45 \\
\hline Calopogonium mucunoides Desv. & 100 & 25.00 & 25.0 & 2.22 & 2.33 & 1.55 & 6.10 & 0.03 & 0.02 & 0.25 \\
\hline Mitracarpus vilosus SW & 50 & 15.00 & 30.0 & 1.11 & 1.40 & 1.86 & 4.37 & 0.03 & 0.02 & 0.60 \\
\hline Pennisetum violaceum (Lam) L. Rich. & 75 & 2.50 & 3.3 & 1.67 & 0.23 & 0.20 & 2.10 & 0.02 & 0.01 & 0.04 \\
\hline Solenostemo monstachyus (P. Beauv) & 75 & 2.50 & 3.3 & 1.67 & 0.23 & 0.20 & 2.10 & 0.02 & 0.01 & 0.04 \\
\hline Asystasia gagentica (Linn) T. Anders & 100 & 17.50 & 17.5 & 2.22 & 1.63 & 1.08 & 4.93 & 0.03 & 0.02 & 0.18 \\
\hline Alternanthera pungens $\mathrm{H} . \mathrm{B}$.and $\mathrm{K}$ & 50 & 7.50 & 15.0 & 1.11 & 0.70 & 0.93 & 2.74 & 0.02 & 0.01 & 0.30 \\
\hline Eragrostic tenella (Linn) P. Beauv. ex Poem and Schult & 100 & 31.25 & 31.25 & 2.22 & 2.91 & 1.93 & 7.06 & 0.04 & 0.02 & 0.31 \\
\hline Perotis indica (Linn) O. Ktze & 50 & 12.50 & 25.0 & 1.11 & 1.16 & 1.55 & 3.82 & 0.02 & 0.01 & 0.50 \\
\hline Paspalum conjugatum Berg & 50 & 7.50 & 15.0 & 1.11 & 0.70 & 0.93 & 2.74 & 0.02 & 0.01 & 0.30 \\
\hline Oplismenus burmanii (Retz) P. Beauv & 50 & 20.00 & 40.0 & 1.11 & 1.86 & 2.48 & 5.45 & 0.03 & 0.02 & 0.80 \\
\hline Pentodon pentandrus Schum and Thonn & 50 & 3.75 & 7.5 & 1.11 & 0.35 & 0.46 & 1.92 & 0.01 & 0.01 & 0.15 \\
\hline Kyllinga bulbosa Beauv. & 50 & 7.50 & 15.0 & 1.11 & 0.70 & 0.93 & 2.74 & 0.02 & 0.01 & 0.30 \\
\hline Gomphrena celosioides Mart. & 50 & 20.00 & 40.0 & 1.11 & 1.86 & 2.48 & 5.45 & 0.03 & 0.02 & 0.80 \\
\hline Spilanthes filicaulis Schum and Thonn & 100 & 11.25 & 11.25 & 2.22 & 1.05 & 0.70 & 3.97 & 0.02 & 0.01 & 0.11 \\
\hline Imperata cylindrical Linn. & 50 & 15.00 & 30.0 & 1.11 & 1.40 & 1.86 & 4.37 & 0.03 & 0.02 & 0.60 \\
\hline Spermacoce verticillata Linn. & 75 & 25.00 & 33.3 & 1.67 & 2.33 & 2.06 & 6.06 & 0.03 & 0.02 & 0.44 \\
\hline Oldenlandia herbacea (Linn) Roxb. & 50 & 50.00 & 100.0 & 1.11 & 4.65 & 6.19 & 11.95 & 0.06 & 0.03 & 2.00 \\
\hline Diodia scandens SW & 50 & 6.25 & 12.5 & 1.11 & 0.58 & 0.77 & 2.46 & 0.02 & 0.01 & 0.25 \\
\hline Eragrostis ciliaria (Linn) R.B. & 50 & 15.00 & 30.0 & 1.11 & 1.40 & 1.86 & 4.37 & 0.03 & 0.02 & 0.60 \\
\hline Digitria horizontalis Willd. & 50 & 7.50 & 15.0 & 1.11 & 0.70 & 0.93 & 2.74 & 0.02 & 0.01 & 0.30 \\
\hline Chloris pilosa Schumach & 100 & 7.50 & 7.5 & 2.22 & 0.70 & 0.46 & 3.38 & 0.02 & 0.01 & 0.08 \\
\hline Phyllantus nururoides Mull-Arg. & 75 & 20.00 & 26.7 & 1.67 & 1.86 & 1.65 & 5.18 & 0.03 & 0.02 & 0.36 \\
\hline Triumfetta cordifolia A. Rich. & 50 & 3.75 & 7.5 & 1.11 & 0.35 & 0.46 & 1.92 & 0.01 & 0.01 & 0.15 \\
\hline Axonopus compressus (SW) P. Beauv. & 100 & 50.00 & 50.0 & 2.22 & 4.65 & 3.10 & 9.97 & 0.05 & 0.03 & 0.50 \\
\hline Eclipta alba (L.) Hassk & 50 & 6.25 & 12.5 & 1.11 & 0.58 & 0.77 & 2.46 & 0.02 & 0.01 & 0.25 \\
\hline Fimbristylis littovalis Gaudet & 50 & 20.00 & 40.0 & 1.11 & 1.86 & 2.48 & 5.45 & 0.03 & 0.02 & 0.80 \\
\hline Paspalum scrobiculatum Linn & 50 & 7.50 & 15.0 & 1.11 & 0.70 & 0.93 & 2.74 & 0.03 & 0.02 & 0.30 \\
\hline Desmodium triflorum (Linn) DC & 75 & 25.00 & 33.3 & 1.67 & 2.33 & 2.06 & 6.06 & 0.03 & 0.02 & 0.44 \\
\hline Scoparia dulcis Linn & 75 & 20.00 & 26.7 & 1.67 & 1.86 & 1.65 & 5.18 & 0.03 & 0.02 & 0.36 \\
\hline Chamaecrista mimosoides (L.) Greene. & 50 & 3.75 & 7.5 & 1.11 & 0.35 & 0.46 & 1.92 & 0.01 & 0.01 & 0.15 \\
\hline Sporobulus pyramidalis P. Beauv. & 50 & 2.50 & 5.0 & 1.11 & 0.23 & 0.31 & 1.65 & 0.01 & 0.01 & 0.10 \\
\hline Bidens pilosa Linn. & 50 & 6.25 & 12.5 & 1.11 & 0.58 & 0.77 & 2.46 & 0.02 & 0.01 & 0.25 \\
\hline Cyperus difformis Linn. & 75 & 25.00 & 33.3 & 1.67 & 2.33 & 3.06 & 6.06 & 0.03 & 0.02 & 0.44 \\
\hline Cyperus esculentus Linn & 50 & 11.25 & 22.5 & 1.11 & 1.05 & 1.39 & 3.55 & 0.02 & 0.01 & 0.45 \\
\hline Cyperus haspan Linn. & 75 & 12.50 & 16.7 & 1.67 & 1.16 & 1.03 & 3.86 & 0.02 & 0.01 & 0.22 \\
\hline Cyperus longibracteatus Cherm. & 75 & 12.50 & 16.7 & 1.67 & 1.16 & 1.03 & 3.86 & 0.02 & 0.01 & 0.22 \\
\hline Melochia mellisifolia (Sm) Jacq & 50 & 11.25 & 22.5 & 1.11 & 1.05 & 1.39 & 3.55 & 0.02 & 0.01 & 0.45 \\
\hline Chromoleana odorata (L) R.M King and Robinson & 75 & 7.50 & 10.0 & 1.67 & 0.70 & 0.62 & 2.99 & 0.02 & 0.01 & 0.13 \\
\hline Desmodium Linn. (Sw) Desv. & 50 & 20.00 & 40.0 & 1.11 & 1.86 & 2.48 & 5.45 & 0.03 & 0.02 & 0.80 \\
\hline Oldenlandia corymbosa Linn. & 100 & 25.00 & 25.0 & 2.22 & 2.33 & 1.55 & 6.10 & 0.03 & 0.02 & 0.35 \\
\hline Phyllantus amarus Schum and Thonn & 50 & 15.00 & 30.0 & 1.11 & 1.40 & 1.86 & 4.37 & 0.03 & 0.02 & 0.60 \\
\hline Sida acuta Burm. F & 50 & 7.50 & 15.0 & 1.11 & 0.70 & 0.93 & 2.74 & 0.02 & 0.01 & 0.30 \\
\hline Sida cordifolia Linn & 50 & 7.50 & 15.0 & 1.11 & 0.70 & 0.93 & 2.74 & 0.02 & 0.01 & 0.30 \\
\hline
\end{tabular}


Table 2: Continue

\begin{tabular}{|c|c|c|c|c|c|c|c|c|c|c|}
\hline Species & $\mathrm{F}(\%)$ & $\mathrm{D}$ & A & $\mathrm{RF}(\%)$ & $\mathrm{RD}(\%)$ & RA (\%) & IVI & SDR & SDE & $\mathrm{A} / \mathrm{F}$ \\
\hline Malvastrum coromandelianum (Linn) Garck & 50 & 5.00 & 10.0 & 1.11 & 0.47 & 0.62 & 2.20 & 0.02 & 0.01 & 0.20 \\
\hline Sida corymbosa R. E. Fries. & 50 & 20.00 & 40.0 & 1.11 & 1.86 & 2.48 & 5.45 & 0.03 & 0.02 & 0.80 \\
\hline Centrosema pubescens Benth & 100 & 7.50 & 7.5 & 2.22 & 0.70 & 0.46 & 3.38 & 0.02 & 0.01 & 0.60 \\
\hline Pycreus lanceolatus (pior) C.B.Cl. & 75 & 30.00 & 40.0 & 1.67 & 2.79 & 2.48 & 6.94 & 0.04 & 0.02 & 0.53 \\
\hline \multirow[t]{2}{*}{ Heterotis rotundifolia (Sm) Jacq. } & 75 & 50.00 & 66.7 & 1.67 & 4.65 & 4.13 & 10.45 & 0.05 & 0.03 & 0.89 \\
\hline & 4500 & 1075.00 & 1615.0 & & & 300.05 & & & & \\
\hline
\end{tabular}

F: Frequency, D: Density, A: Abundance, RF: Relative frequency, RD: Relative density, IVI: Importance value index, SDR: Species diversity richness, SDE: Species diversity evenness, A/F: Abundance to frequency ratio

\section{DISCUSSION}

Phytodiversity studies in tropical rainforest are important to determine the processes or mechanisms that maintain high diversity, species richness, species assemblages and at the same time providing a database about the number and status of the species existing in an area and their conservation ${ }^{37,38}$. Pollution has been shown to reduce plant species diversity, simplify community structure, lower productivity and caused nutrient imbalance in many ecosystem ${ }^{39}$.

The effect of oil spill in a fresh water marsh has been studied to ascertain the diversity of vegetation, total plant cover, total species number, mean number of species per quadrate in both high and mid marsh zones after some years of spillage leading to differences in final and initial species count $t^{40}$. Similar study of hydrocarbon disturbance on species count could also be associated with the post remediated Ahia oil-field. The existence of species in the study area largely depends also on its regeneration under varied local environmental conditions. Upon such condition open canopy might favour the vegetation establishment through increased solar radiation incident on the forest floor. Consequently this could also influence the growth stages in seedling, sapling and young shrubs, herbs and trees of plant communities that maintain the population structure of the vegetation of the area. This corroborates the assertion that open canopy may favour germination and seedling establishment through increased solar radiation on forest floor $^{41,42}$.

Regeneration is a critical phase of forest management, because it maintains the desired species composition and stocking after disturbance ${ }^{13}$. This may also be true for the present research at Ahia oil field. Many species at the various stations which supposedly under natural habit are trees were not present as trees in the study site. So, new species were found regenerating, with many exhibiting multiplier effect through coppicing, seedling/sapling and rhizomes in their mode of regeneration status. The quantitative structure of regeneration in the study area also reveals the biometric ecology of the study site. The study area was found to be dominated by the seedlings/saplings of Oldenlandia herbacea, Axonopus compressus and
Heterotis rotundifolia. However, among the recorded species, the dominant species are not only important for biodiversity conservation but also of commercial importance in ethno-botany and ethno-medicine.

The usefulness of phytosociological parameters in determining the status of different tropical forest ecosystems in terms of floristic composition, species diversity and forest structure has been highlighted by several works ${ }^{37,38,43-45}$. These studies independently confirmed the floristic diversity and richness of tropical forest ecosystems in terms of number of species, genera and families and defined vegetation structure which are all reported for the Ahia oil field in the study area. Similar index of study has been revealed among the prevalent Taxa in the study site in which Poaceae had the highest richness of species with $A$. compressus recording the highest diversity in richness and evenness.

Species abundance distribution is one of the few universal patterns in ecology. Research on this fundamental distribution has primarily focused on the study of numerical counts, irrespective of the traits of individuals. It has been suggested that investigating abundance measures other than number of individual may lead more direct to mechanistic in ecological research $^{46}$. The greater abundance of lower vascular species among the families is an indication of a secondary vegetation structure heterogeneous in nature as a result of the regeneration process with new species that were absent as adult. The presence of such secondary forest species as shown in the Table 2 in this study site points towards anthropogenic incursions at different times of oil spillage into the pristine forest of the Ahia oil field, resulting to differences in the ranks of species frequency, diversity (richness and evenness), density and abundance. This corroborates the assertion that as anthropogenic gaps into natural forest become numerous, the landscape pattern and species composition of that forest changes gradually ${ }^{43}$. Study has shown that perennial species generally have less impact than annual species in a post spill incident in the analysis of species abundance. Khater et al. ${ }^{47}$ compared levels of degradation and the resulting species communities and it was found that less degraded sites favoured woody shrubs and perennial herbs while more degraded site 
favored annual plants. Similar life form of herbaceous habit has also been documented in post-impacted and remediated site in parts of Akwa-Ibom State, Nigeria ${ }^{3}$.

The present study shows that the study area can be brought under complete forest by natural regeneration of forest establishment. Complete absence of seedlings and sapling indicates poor regeneration, while presence of population indicates successful regeneration ${ }^{48}$. The density, frequency, abundance, species diversity, Relative Diversity (RD), Relative Frequency (RF) and Relative Abundance (RA) considered as indices of success in reforestation ${ }^{49}$ as well as the greater proportion of the species exhibiting multiplier mode of regeneration suggests that it is possible to re-establish a complete forest cover for the degraded deforested Ahia oil field area by natural regeneration. The recorded indices are considered quite adequate to establish complete forest cover, a very important step for biodiversity conservation. Ratio of abundance to frequency (A/F) indicates that the distribution of all the regenerated species was contiguous. In a similar assertion, contiguous distribution pattern is the prevalent pattern in nature unlike random distribution found in very uniform environments. Contiguous distribution in natural vegetation has been reported by several workers ${ }^{3,4,50}$. From the research findings, it is evident that the oil impacted post-remediated forests of Ahia oil field are turning into diverse heterogeneous natural forest again.

\section{CONCLUSION}

The findings of present study provide a complete view of regeneration status in the study area, which is rich in regenerating species that could result in the establishment of a diverse natural forest if protected to conserve the seedlings or saplings of the regenerating species. The result shows that it is possible to bring the depleted area under complete forest cover through the protection of natural regeneration.

\section{REFERENCES}

1. Cubizolle, H., A. Tourman, J. Argant, J. Porteret, C. Oberlin and K. Serieyssol, 2003. Organs of European biodiversity: Palaeo-geographic signification of peat inception during the Holocene in the granite Eastern Massif Central (France). Landscape Ecol., 7: 211-227.

2. Whitfield, D.P., D.R.A. McLeod, A.H. Fielding, R.A. Broad, R.J. Evans and P.F. Haworth, 2007. The effects of forestry on golden eagles on the island of Mull, western Scotland. Western Scotland J. Applied Ecol., 38: 1208-1220.
3. Edwin-Wosu, N.L. and E.A.B. Edu, 2013. Eco-taxonomic assessment of plant species regeneration status in a post-remediated crude oil impacted site in parts of Ibibio-I-Oil field in Ikot-Ada Udo, Ikot-Abasi Local Government Area of Akwa Ibom State, Nigeria. Asian J. Plant Sci. Res., 3: 14-23.

4. Edwin-Wosu, N.L., E.A.B. Edu and O.M. Okeke, 2013. Floristic and diversity trend of regeneration in a quartz dominated quarry impacted site in parts of Umuoke, in Obowo local government area of Imo State, Nigeria. Asian J. Plant Sci. Res., 3: 142-156.

5. Edwin-Wosu, N.L. and E.N. Elenwo, 2006. Crude oil exploration-an environmental double-edged sword in Nigeria: The Niger delta experience. J. Nigeria Environ. Soc., 3: 268-279.

6. Kinako, P.D.S., 1981. Mathematical elegance and ecological naivety of diversity indices. Bull. Sci. Assoc. Nig., 7: 39-42.

7. Anderson, G., 2005. Niger Delta Basin: A Vision for Sustainable Development. The World Bank, Washington, DC., USA., Pages: 131.

8. Wilcox, C.A., Y.M. Chun and Y.D. Choi, 2005. Redevelopment of black oak (Quercus velutina Lam.) savanna in an abandoned sand mine in Indiana dunes national lakeshore, USA. Am. Midland Nat., 154: 11-27.

9. Yuan, J.G., W. Fang, L. Fan, Y. Chen, D.Q. Wang and Z.Y. Yang, 2006. Soil formation and vegetation establishment on the cliff face of abandoned quarries in the early stages of natural colonization. Restoration Ecol., 14: 349-356.

10. Duan, W.J., H. Ren, S.L. Fu, J. Wang, L. Yang and J.P. Zhang, 2008. Natural recovery of different areas of a deserted quarry in South China. J. Environ. Sci., 20: 476-481.

11. Novak, J. and K. Prach, 2003. Vegetation succession in basalt quarries: Pattern on a landscape scale. Applied Vegetation Sci., 6: 111-116.

12. Lyngdoh, T., R.S. Tripathi and A.K. Das, 1992. Vegetation dynamics on coal mine spoils of Jaintia hills in Meghalaya(north-east India) undergoing natural recovery. Acta Oecologica-Int. J. Ecol., 13: 767-776.

13. Duchok, R., K. Kent, A.D. Khumbongmayum, A. Paul and M.L. Khan, 2005. Population structure and regeneration status of medicinal tree Illicium griffithii in relation to disturbance gradients in temperate broad-leaved forest of Arunachal Pradesh. Curr. Sci., 89: 673-676.

14. Ashton, P.S. and P. Hall, 1992. Comparisons of structure among mixed dipterocarp forests of North-western Borneo. J. Ecol., 80: 459-481. 
15. Hopkin, B., 1968. Vegetation of the Olakemeji forest reserve. Nig. J. Ecol., 56: 97-115.

16. SAF., 1954. Forest Cover Types of North America. Society of American Foresters, Washington, DC., USA.

17. Edwin-Wosu, N.L., 2010. Eco-taxonomic baseline assessment of vegetation of pirigbene-obama-agip oil field further development project in Southern Ijaw local government area, Bayelsa State. Final Draft Report, NAOC/Sydney Gate-way (Nig) Limited, October 2010, pp: 3-38.

18. Edwin-Wosu, N.L., 2011. Eco-taxonomic post-impact assessment of vegetation of GOI lake oil spillage (EIA SHEWING). Vegetation Survey Claim of GOI Community Against Shell Petroleum Development Company (SPDC) in Gokana Local Government Area, Gokana, Rivers State, Nigeria, pp: 3-19.

19. Edwin-Wosu, N.L., 2012. Environmental evaluation study of vegetation of the disused imo river. Nkpoku, Obigbo North, Ebubu Pipeline (ROW) in Rivers State/and Abia State, MACPHED/SPDC, Wet Season Draft Report, February, 2012, pp: 1-37.

20. Edwin-Wosu, N.L., 2012. EIA baseline study of vegetation/wildlife for the OBIAFU/OBRIKOMIndorama Eleme Fertilizer Company Limited (IEFCL) gas delivery pipeline project. Environmental and Chemical Services Limited, Idorama Eleme Petrochemical Company Limited, Nigeria, August 2012, pp: 1-90.

21. Kinako, P.D.S., 1988. Fundamental of Quantitative and Applied Plant Ecology. Belk Publishers, USA., pp: 26-45.

22. Hutchinson, J. and J.M. Dalziel, 1954. Flora of West Tropical Africa. Vol. 1, Part 1, Crown Agents for Oversea Governments and Administrations, London, UK., pp: 32-295.

23. Hutchinson, J. and J.M. Dalziel, 1958. Flora of West Tropical Africa, Vol. 1, Part II, Crown Agents for Oversea Governments and Administrations, London, UK., pp: 298-756.

24. Hutchinson, J.B. and J.M. Dalziel, 1963. Flora of West Tropical Africa. Vol. 2, Crown Agents for Oversea Governments and Administration, London, UK., Pages: 544.

25. Hutchinson, J., J.M. Dalziel, 1968. Flora of West Tropical Africa. 2nd Edn., Vol. 3, Crown Agents for Overseas Government and Administration, London, UK., pp: 5-276.

26. Hutchinson, J. and J.M. Dalziel, 1972. Flora of West Tropical Africa. 2nd Edn., Vol. 3, Crown Agents for Overseas Government, London, UK., pp: 277-512.
27. Joyce, L. and D.P. Stanfield, 1974. The Flora of Nigeria Sedges (Cyperaceae). Ibandan University Press, Ibadan, Nigeria, Pages: 140.

28. Joyce, L., 1989. Flora of Nigeria Grasses. Ibadan University Press, Ibadan, Nigeria, pp: 28-294.

29. Austin, M.P. and P. Greig-Smith, 1968. The application of quantitative methods to vegetation survey: II. some methodological problems of data from rain forest. J. Ecol., 56: 827-844.

30. Kershaw, K.A., 1973. Quantitative and Dynamic Plant Ecology. Edward Arnold Ltd., London, UK., Pages: 308.

31. Shukla, S.R. and S.P. Chandal, 1980. Plant Ecology. 4th Edn., SCCR, New Delhi, Pages: 197.

32. Bonham, C.D., 1989. Frequency: Measurement of Terrestrial Vegetation. John Wiley and Sons, New York, USA., pp: 90-96.

33. Shannon, C.E. and W. Weaver, 1963. The Mathematical Theory of Communications. University of Illinois Press, Urbana, USA., pp: 367.

34. Pryor, L.D., 1981. Australian endangered species: Eucalyptus. Australian National Parks and Wildlife Service's Special Publication Australia Canberra, pp: 1-139.

35. Misra, R., 1968. Ecology Workbook. Oxford and IBH Publishing Co. Ltd., New Delhi, India, Pages: 244.

36. Curtis, J.T. and G. Cottam, 1956. Plant Ecology Work Book: Laboratory Field Reference Manual. Burgess Publishing Co., Minnesota, pp: 193.

37. Prasad, P.R.C., C.S. Reddy and C.B.S. Dutt, 2007. Phytodiversity assessment of tropical rainforest of North Andaman Islands, India. Res. J. Forestry, 1: 27-39.

38. Reddy, C.S., S. Babar, A. Giriraj, K.N. Reddy and K.T. Rao, 2008. Structure and floristic composition of tree diversity in tropical dry deciduous forest of Eastern Ghats, Southern Andhra Pradesh, India. Asian J. Scient. Res., 1: 57-64.

39. Whittaker, R.H. and G.M. Woodwell, 1970. Structure, production and diversity of the Oakpine forest at Brookhaven, New York. Ecology, 57: 155-174.

40. Burk, C.J., 1977. A four year analysis of vegetation following an oil spill in a freshwater marsh. J. Applied Ecol., 14: 515-522.

41. Khan, M.L., J.P.N. Rai and R.S. Tripathi, 1987. Population structure of some tree species in disturbed and protected sub-tropical forests of northeast India. Acta Oecol. Oecol. Applicata, 8: 247-255. 
42. Srinivas, C., 1992. Plant biomass, net primary productivity and nutrient cycling in oak (Quercus serrata Thumb). Ph.D. Thesis, Forests of Manipur, Manipur University, Manipur, India.

43. Young, S. and L.N. Swiacki, 2006. Surveying the forest biodiversity of Evansburg State Park: Plant community classification and species diversity assessment. Int. J. Bot., 2: 293-299.

44. Sahu, S.C., N.K. Dhal, C.S. Reddy, C. Pattanaik and M. Brahmam, 2007. Phytosociological study of tropical dry deciduous forest of boudh district, Orissa, India. Res. J. Forestry, 1: 66-72.

45. Agbagwa, I.O., 2008. Impact of the construction of access roads to oil well locations and flowstations on the phytodiversity of some Niger delta floodplains. J. Applied Sci. Res., 4: 1876-1884.

46. McGill, O., P.B.A.N. Kumar, V. Dushenkov, H. Motto and I. Raskin, 2007. Taking species abundance distribution beyond individuals. Department of Ecology, Faculty of Science, Charles University, Prague Czech Republic.
47. Khater, C., A. Martin and J. Maillet, 2003. Spontaneous vegetation dynamics and restoration prospects for limestone quarries in Lebanon. Applied Vegetation Sci., 6: 199-204.

48. Saxena, A.K., S.P. Singh and J.S. Singh, 1984. Population structure of forests of Kumaun Himalaya: Implications of management. J. Environ. Manage., 19: 307-324.

49. Rajwar, G.S., M. Dhaulakhandi and P. Kumar, 1999. Regeneration status of an Oak forest of Garhwal Himalaya. Indian For., 125: 623-630.

50. Verma, R.K., D.K. Shadangi and N.G. Totey, 1999. Species diversity under plantation raised on a degraded land. Malaysian For., 62: 95-106. 